\title{
Solar Rejection Window and Narrow Bandpass Filters for the Meteosat Third Generation Lightning Imager
}

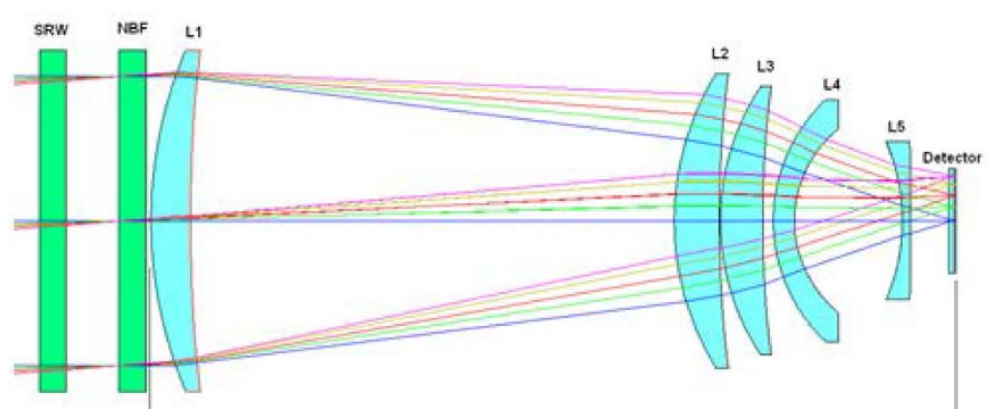

ICSO 2018, Chania Greece, 12-Oct-2018

Presented by Dr. Claude Montcalm
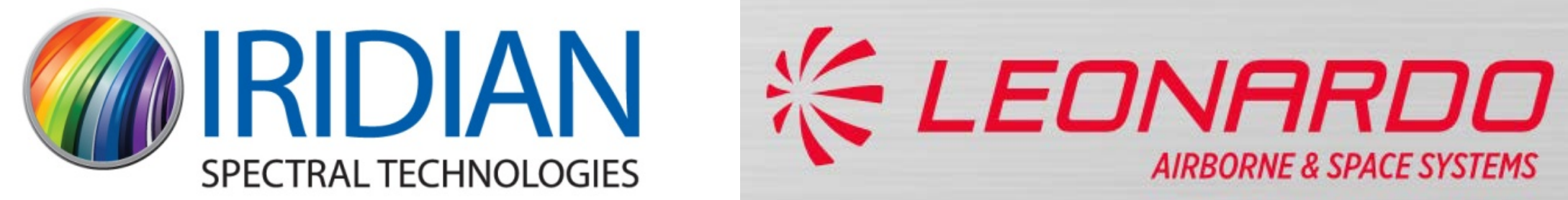


\section{Outline}

- MTG LI

- Goals, requirements, LI optical design

- Solar Rejection Window (SRW) and Narrow Bandpass Filter (NBF)

- Functions and requirements

- Optical interference filters design considerations

- Filter fabrication and characterization

- Deposition and measurements

- Adjustment of NBF by annealing

- At-wavelength TWE verification

- Durability verification

- Summary
Iridian authors:

- Claude Montcalm

- Adam Badeen

- Douglas Burbidge

- Robert Bruce

- Graham Carlow

- Justin Dane

- Nadim Firdawsi

- George E. Laframboise

- Alex M. Miles

- Jean-Paul Noel

- Robert Rinfret

- Brian T. Sullivan

Leonardo authors:

- Stefano Lorenzini

- Riccardo Bardazzi

- Lorenzo Giunti 


\section{MTG-Lightning Imager}

- MTG objective: provide Europe \& International Community with accurate prediction of meteorological phenomena and monitoring of climate and air composition.

- Lightning Imager (LI) is one of the instruments on the MTG-I satellites

- It provides a real time location of lightning events by detection of $\mathrm{O}_{2}$ discharge triplet at $777.4 \mathrm{I}-777.75 \mathrm{~nm}$

- 2 nm spectral bandwidth

- Ims temporal sampling

- spatial resolution (GSD) $4.5 \mathrm{~km}$ at sub-satellite point

- optical pulse dynamic range 6.7 to $670 \mathrm{~mW} / \mathrm{m}^{2} / \mathrm{sr}$

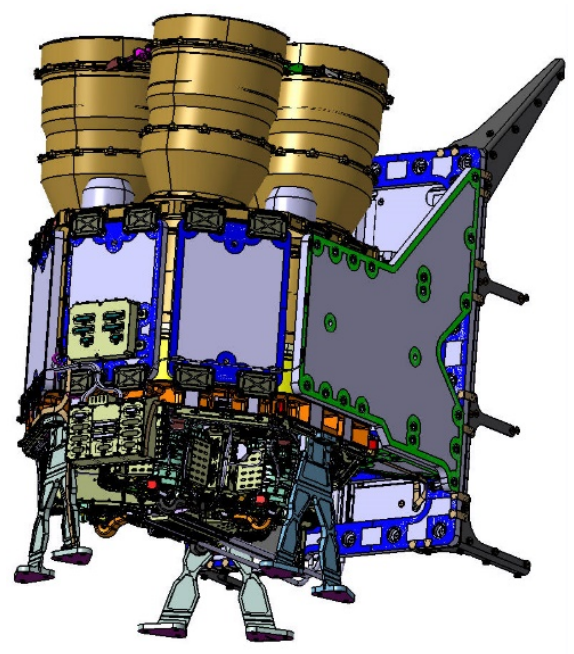

- Launch of four MTG imaging satellites starting from 202I onwards, with 20 years of overall operational services expected

- Thales Alenia Space - France is the MTG prime contractor; Leonardo is the LI mission prime; Iridian provides the SRW and NBF filters discussed here. 


\section{Lightning Imager (LI) Instrument Overview}

- The $\mathrm{LI}$ is composed of one optical head and one electronic box

- The optical head consists of four identical optical channels, each one including:

- a baffle for stray light suppression and thermal load minimization;

- a SRW to minimize both the background level and the thermal load;

- a NBF to reduce the spectral bandwidth in the range of the lightning spectral pulse;

- an optical system with F\# I.73, II0 mm entrance pupil;

- a CMOS detector (1000x I I 70 pixels, $24 \mu \mathrm{m}$ pitch, 1000 frames/sec digital output);

- a processing electronics implementing the detection functions

- Active thermal control devices insure temperature stabilization of critical components during the operational phase

- The four cameras are arranged to cover more than $84 \%$ of the observable Earth disc

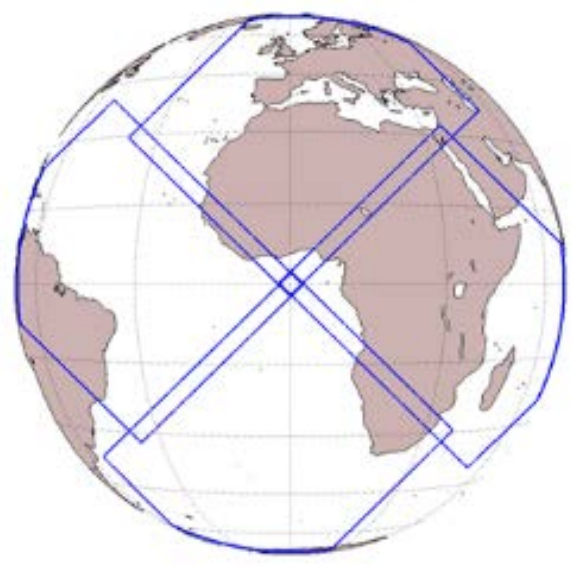




\section{Optical System}

- Each optical channel has an independent single stage lens, detector and baffle.

- The SRW and the NBF $(\varnothing \mid 30 \mathrm{~mm})$ are the first two parallel plates followed by the five lenses.

- The optical system is athermalized in at least the specified thermal range.

- Ensquared energy and transparency are the driver requirements of the optical design to guarantee high detection probability at instrument level.

\begin{tabular}{|cc|}
\hline Parameter & Value \\
\hline Effective Focal length & $190.8 \mathrm{~mm}$ \\
\hline Entrance pupil diameter & $110 \mathrm{~mm}$ \\
\hline Entrance pupil position & on the NBF filter \\
\hline Field of View & $5.1^{\circ}$ \\
Transparency (with filters) & $77 \%$ \\
Wavelength range & $777.41-777.75 \mathrm{~nm}$ \\
Ensquared energy EOL & $90 \%$ \\
Max vignetting & 0 \\
Pixel pitch & $0.024 \mathrm{~mm}$ \\
Thermal range & 20 to $50^{\circ} \mathrm{C}$ \\
\hline
\end{tabular}

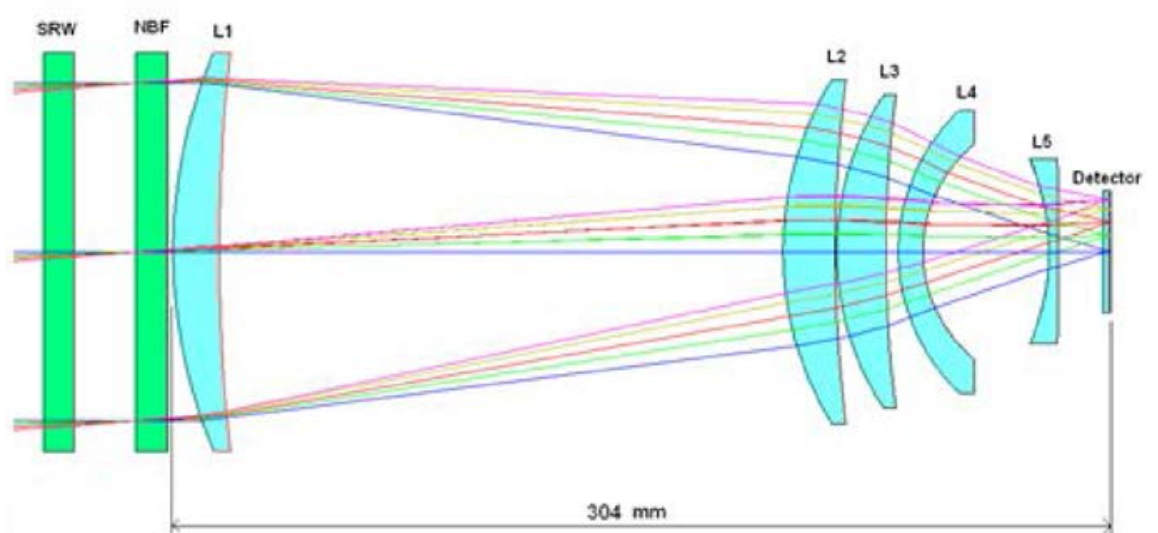




\section{SRW \& NBF combined solar radiation rejection}

- The SRW and NBF work in synergy to obtain the required spectral filtering

\section{SRW and NBF combined optical filtering}

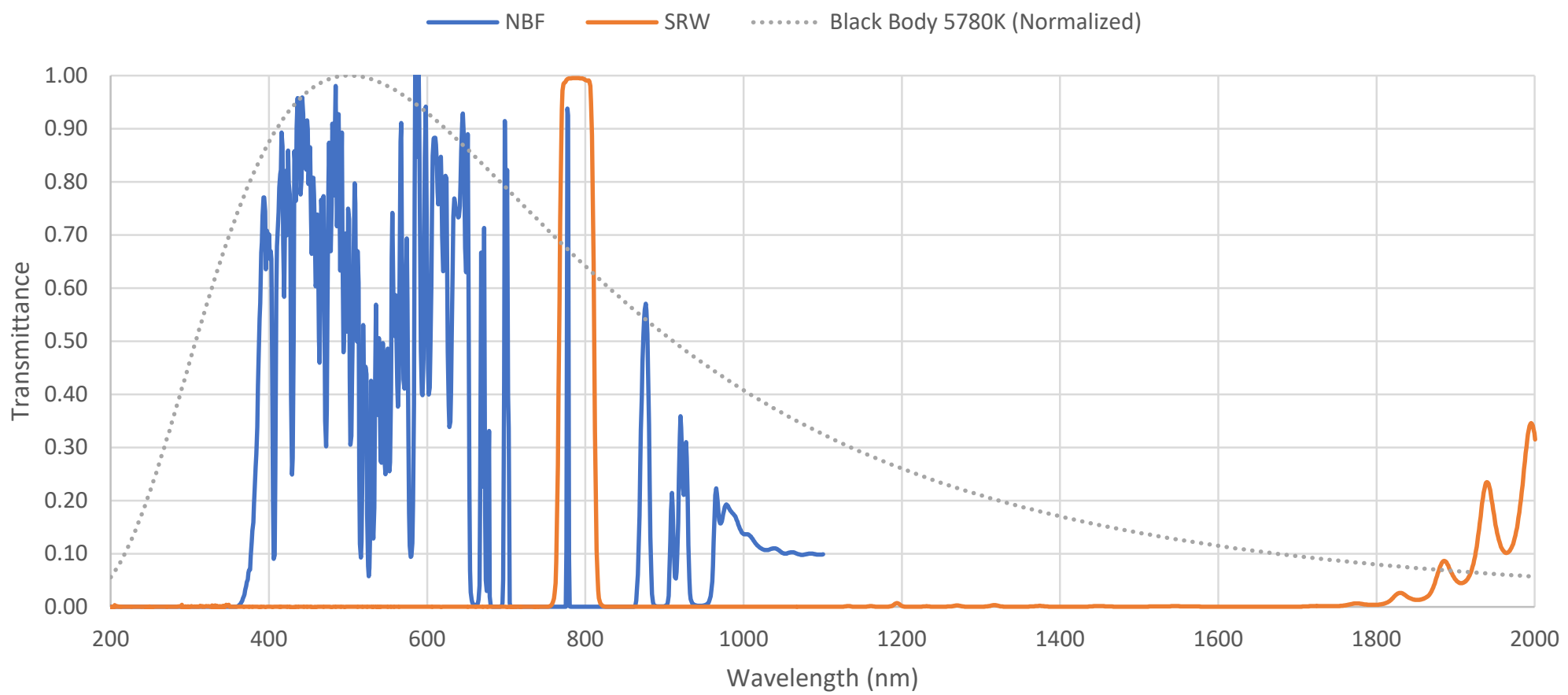

- The SRW is reflecting from UV to IR except from 760 to $810 \mathrm{~nm}$ and the NBF further filters near the $\mathrm{O}_{2}$ triplets $(777.4 \mathrm{I}-777.75 \mathrm{~nm})$ to get the good $\mathrm{s} / \mathrm{n}$. 


\section{SRW design requirements}

- Designed to reflect most of the solar radiation but pass the lightning $\left(\mathrm{O}_{2}\right)$ discharge spectral region ( $760 \mathrm{~nm}$ to $810 \mathrm{~nm})$

- Directly exposed to the external space environment

- Experiences much larger temperature excursions than the rest of the system

- mechanical mounting is thermally decoupled from the rest of the optical system

- Must be radiation resistant (uses Schott BK7GI 8 substrates)

- Optical performance requirements:

- Transmittance $>97 \%$ in the $\mathrm{O}_{2}$ discharge line range

- High reflectance/transmittance ratio of the sun light in the 200 to $2000 \mathrm{~nm}$

- High rejection-absorption in MWIR

- Minimize ghost generation and polarization sensitivity for all angles from 0 to $16.3^{\circ}$ (baffle cut-off angle) 


\section{SRW design considerations}

- The SRW multilayer coating must reflect light over a wide wavelength range and is therefore relatively thick, i.e., $\sim 40 \mu \mathrm{m}$ (metric thickness)

- A two-sided design approach was adopted for the SRW filter

- Balances the coating thickness between the two surfaces

- Reduces surface defects and ensure compliance for the surface figure (curvature)

- The "space" side reflects MWIR and the "instrument" side reflect in the VIS-IR

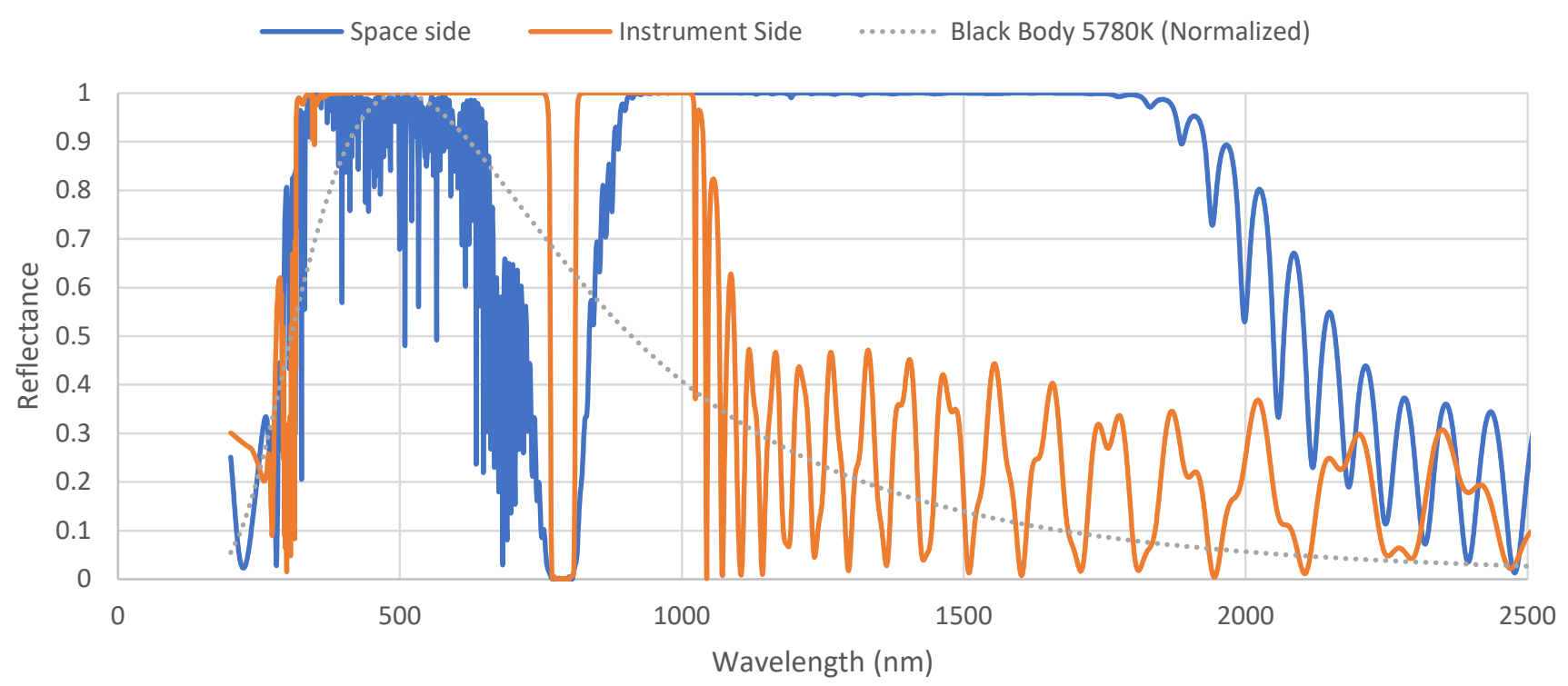

- The drawback to this balanced design approach is that it increases the risk of ghosted images (also beam walk-off at higher angles) 


\section{NBF design requirements}

- NBF is designed to perform the spectral discrimination of the lightning pulse from the Earth background radiance.

- Supported by the lens mounting system so its operative thermal range is the same as the rest of the optical system

- Optimize transmittance over the $\mathrm{O}_{2}$ discharge triplet range (weighted average)

- Minimize the pass band width around the triplet

- Optimization must consider the $\mathrm{AOI}$ and temperature range

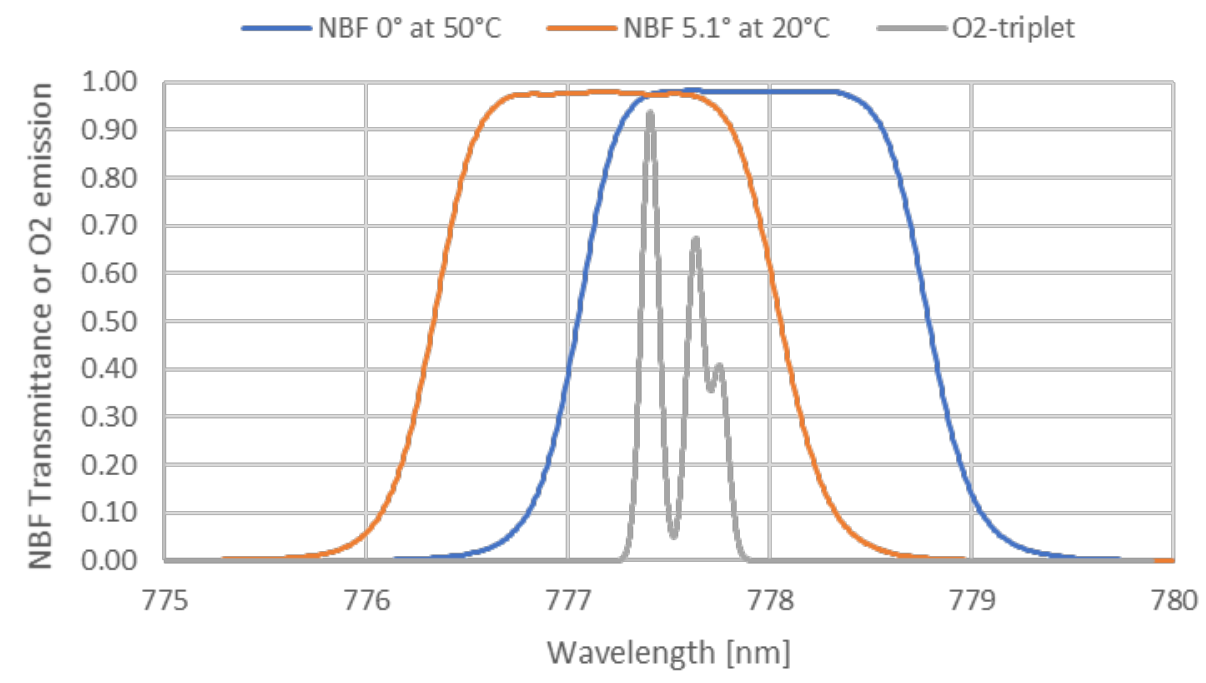

- Also minimize ghost reflection and polarization sensitivity 


\section{NBF design considerations}

- The NBF (space) side design is based on a classical multi-cavity Fabry Perot structure; the other side (instrument) is simple AR coating.

- A complex modeling scheme was developed to track all optical performance requirements

- Analysis showed the max wavelength shift was $0.45 \mathrm{~nm}$, imposing a total thickness uniformity variation $<0.05 \%$ over the clear aperture $(\varnothing \mathrm{I} / 4 \mathrm{~mm})$

- Important tradeoffs were made in favor of $T_{N B F}$ and $E B_{N B F}$ :

- NBF side max reflectance and polarization sensitivity were both increased from initial request

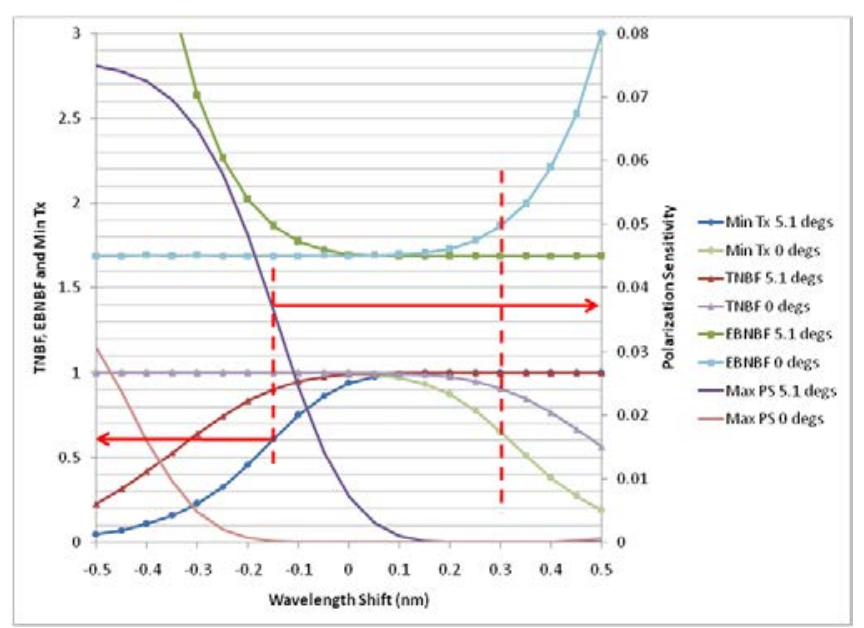




\section{Filter Fabrication}

- Both the SRW and NBF filters are composed of $\mathrm{SiO}_{2} / \mathrm{Ta}_{2} \mathrm{O}_{5}$ multilayers deposited using reactive magnetron sputtering deposition systems equipped with in-situ optical monitoring

- Reactive magnetron sputtering produces $\mathrm{SiO}_{2} / \mathrm{Ta}_{2} \mathrm{O}_{5}$ oxide materials that are very robust and dense (free of porosity)

- Not affected by air to vacuum transition

- Very durable (humidity - temperature, scratch resistant, etc. $\rightarrow$ Telcordia)

- High radiation resistance

- The SRW filters require "typical" uniformity $(<0.3 \%)$ so a multi-substrate planetary coating platform was used

- The NBF required a very good thickness uniformity that required fine-tuning the deposition geometry on a different deposition platform 


\section{SRW Characterization Results}

- A large number of measurements are required to fully qualify the SRW

- $T_{x}$ and $R_{x}$,over full clear aperture (CA), both s- and p-polarization, AOI: 0 to 16.3 Wavelength: 200 to $10,000 \mathrm{~nm}$, Temperature: $-20^{\circ}$ to $+80^{\circ} \mathrm{C}$

- Overall characterization approach:

- Multilayer coating model is built during deposition with in-situ (real time) monitoring

- Full filter area is "mapped" with $T_{u}$ at $0^{\circ} \mathrm{AOI}$ and high resolution to confirm uniformity

- A set of $T_{s / p}$ and $R_{s / p}$ measurements in the center of the filters at various angles confirm model validity
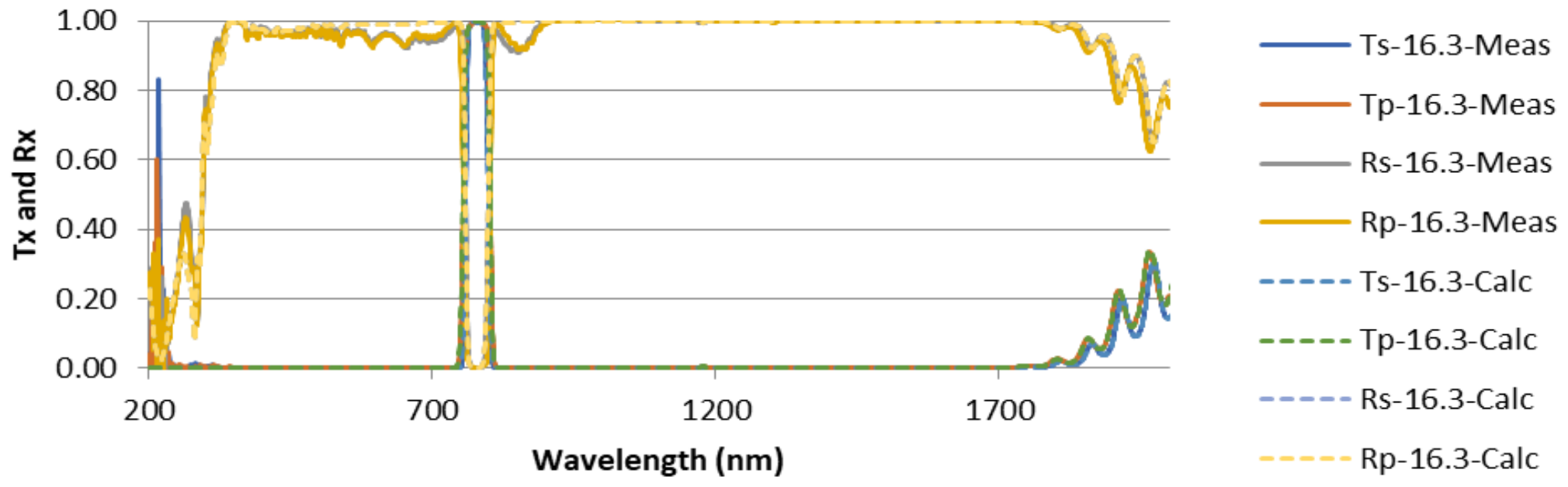

- Measurements and model confirmed accordance with all requirements 


\section{NBF uniformity and optical performance}

- The center wavelength $(C W L)$ variation is $0.014 \%( \pm 0.007 \%)$ inside the clear aperture (radius of $57 \mathrm{~mm}$ )
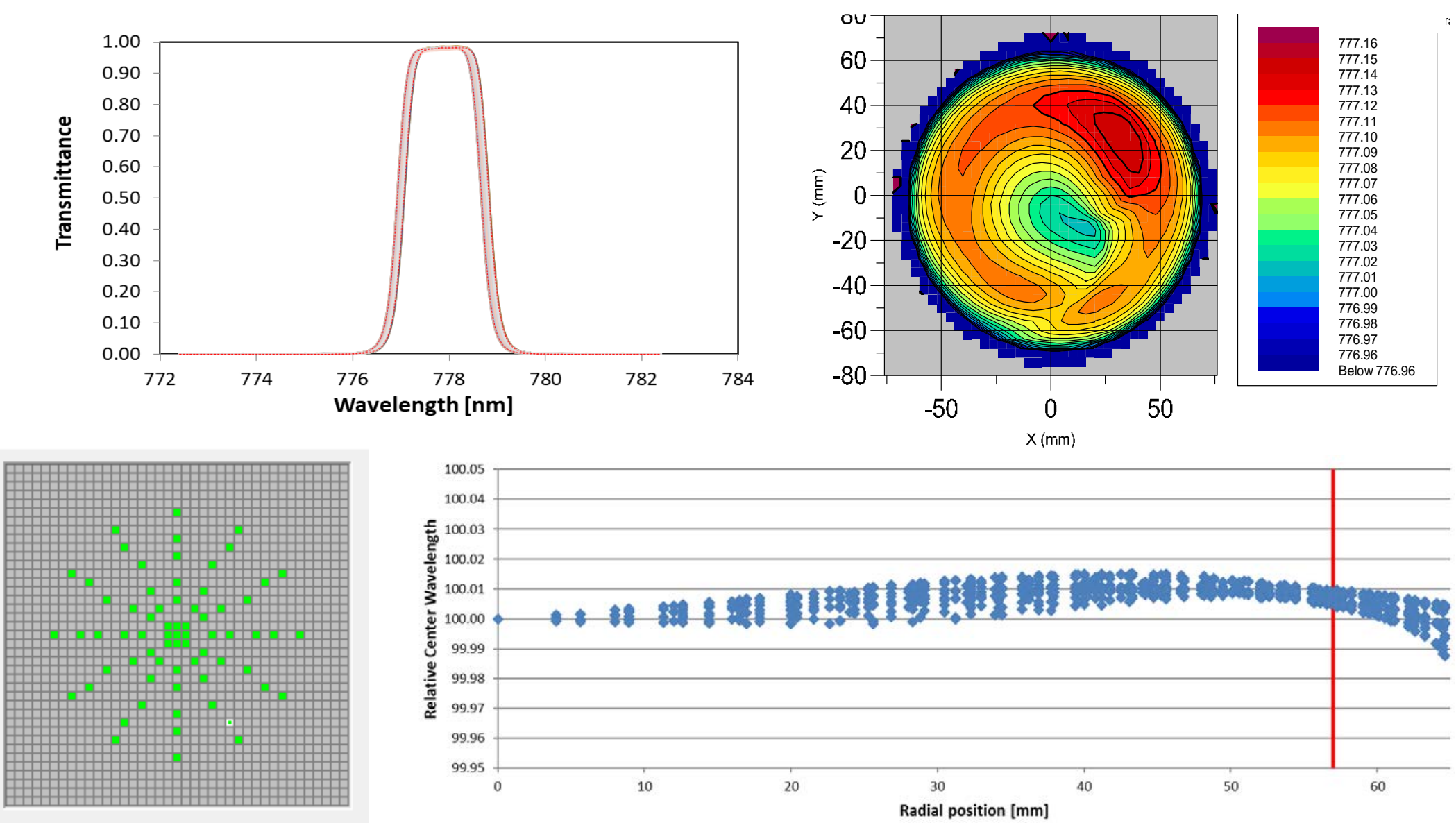


\section{NBF Shift-width analysis}

- Wavelength tolerance, or shift width, is estimated from a subset of 69 measured transmittance curves across the substrate

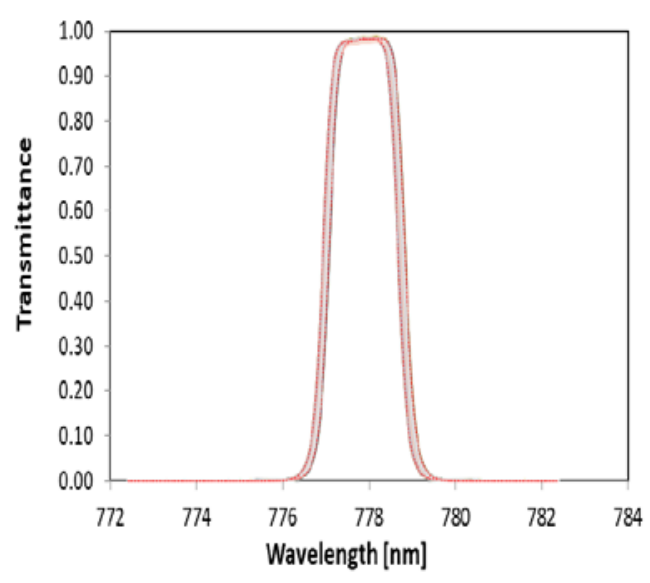

- The entire filter area must pass both $T_{N B F}$ and $E B_{N B F}$ after wavelength shifting due to angle, temperature and life-time aging

- Approach:

I. Find the maximum wavelength down-shift and up-shift possible (based on lowest and highest CWL curves)

2. Reduce margins to account for angular shift at $5.1^{\circ}(-0.762 \mathrm{~nm})$, estimated lifetime aging $(+0.010 \mathrm{~nm})$, and thermal shifts $\left(+3.5 \mathrm{pm} /{ }^{\circ} \mathrm{C}\right)$

3. Determine the optimum wavelength shift so optical requirements for $T_{N B F}$ and $E B_{N B F}$ are met for all 69 measurement points

4. Anneal to target the desired wavelength shift

5. Iterate steps 3 and 4 as required 


\section{Anneal tuning and stabilization}

- $\mathrm{SiO}_{2} / \mathrm{Ta}_{2} \mathrm{O}_{5}$ sputtered oxide coatings age by diffusion mechanisms that are thermally activated

- Changes in thickness and index $\rightarrow$ optical thickness

- Can be modelled by the Arrhenius rate equation $\Delta C W L=f(t) \exp \left(-E_{a} / k T\right)$ where $f(t)$ is a time dependence function that is empirically determined

- With annealing, the NBF filters CWL can increases $(\sim \mathrm{Im})$ and can be tuned to within $\pm 10 \mathrm{pm}$, without change in transmittance level

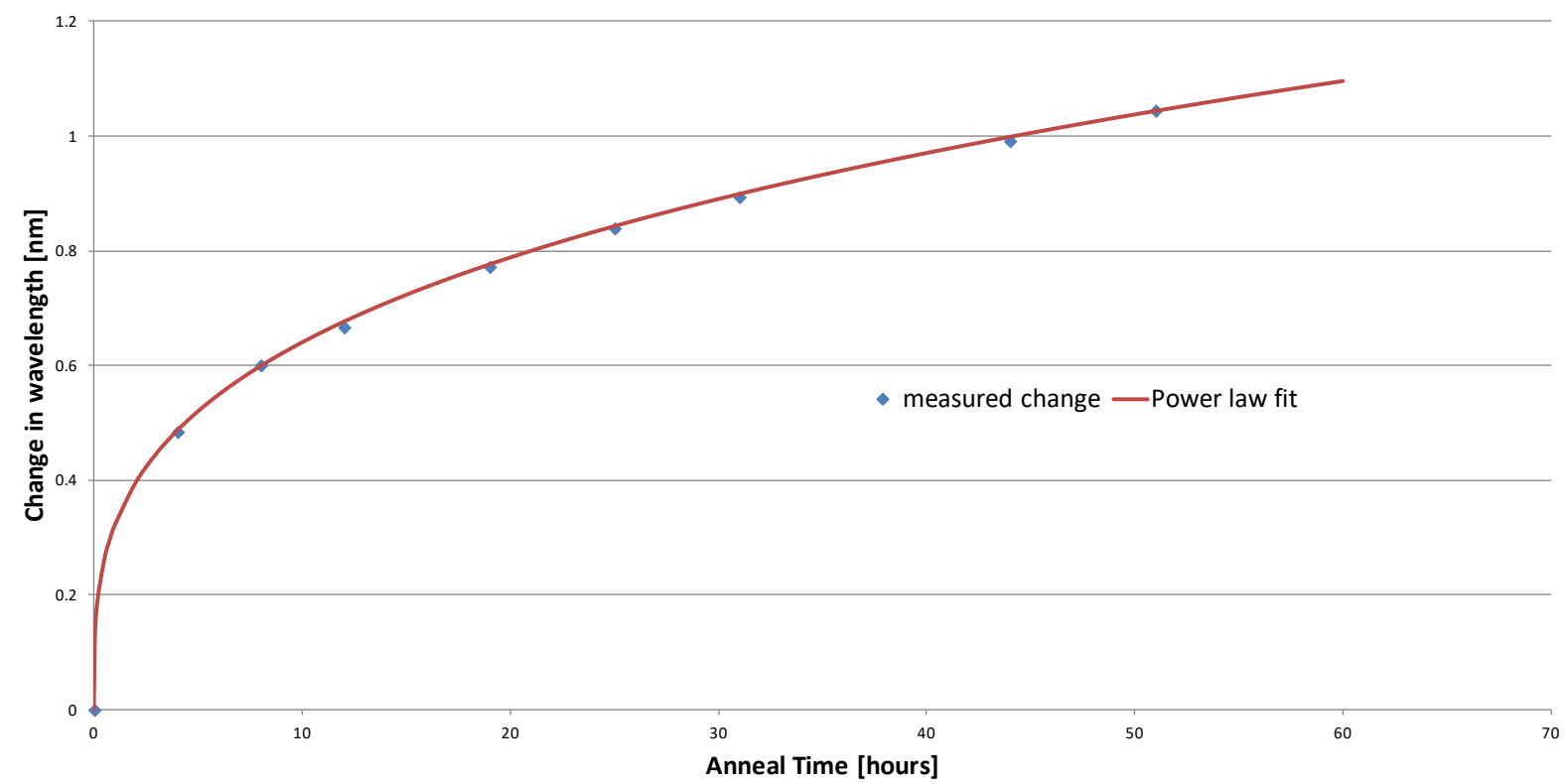




\section{At-wavelength verification of flatness and TWE}

- Most common Fizeau interferometers work at $633 \mathrm{~nm}$ but the NBF/SRW either do not transmit or reflect at this wavelength, preventing TWE or RWE measurements

- Instead, used a multi-wavelength wavefront metrology bench from Phasics based on

- Quadri-wave lateral shearing interferometry, where the wavefront is sampled passing through a modified Hartman mask and recorded on a CCD camera

- Three LED sources $(625,780$, and $1050 \mathrm{~nm})$, SID4-HR sensor (400×300 pix).

- Measured transmitted and reflected wavefront error (TWE and RWE)

- Specifications: TWE <140 nm RMS total, and <25 nm RMS after power subtraction
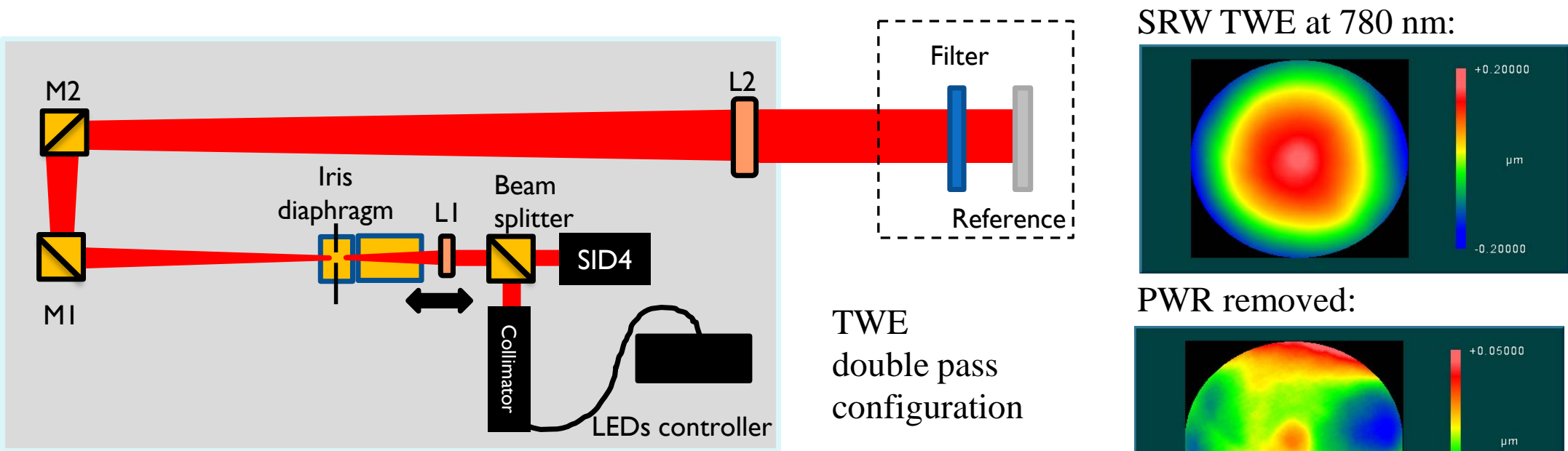

Courtesy of Phasics (www.phasics.fr)

PWR removed:

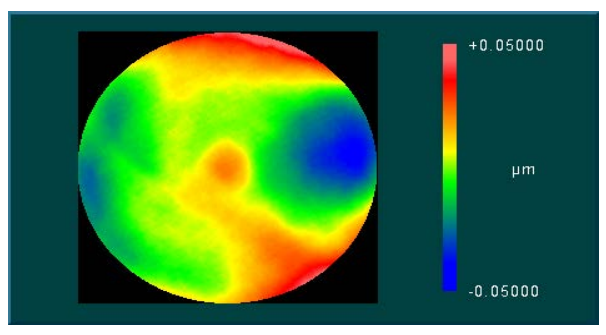




\section{Durability verification}

- Solubility, abrasion, adhesion, and humidity (in-house)

- Vacuum thermal cycling (Integrity Testing Lab)

- $-110^{\circ} \mathrm{C}$ to $+160^{\circ} \mathrm{C}, 30$ min dwell, 30 cycles

- Air-to-vacuum (in-house using a spectrophotometer + cryostat)

- Radiation exposures:

- $2 \mathrm{MeV}$ protons at the Tandetron (University of Western Ontario, Ontario)

- Up to $1.9 \mathrm{E}+13 \mathrm{p}^{+} / \mathrm{cm}^{2}$ (SRW) and $4.1 \mathrm{E}+09 \mathrm{p}^{+} / \mathrm{cm}^{2}$ (NBF)

- Gamma Cobalt60 at Nordion (Laval, Quebec)

- Up to $600 \mathrm{kRad}$ (NBF only)

- Solar UV and electrons at SCEPTER (US Air Force Research Lab, Ohio)

- Up to I500 ESH of combined VUV, UV

- $2 \mathrm{E}+16 \mathrm{e}-/ \mathrm{cm}^{2}$ fluences of $20 \mathrm{keV}$ electron flux 


\section{Summary}

- Four optical channels composes the LI optical head, and where each have a:

- SRW to minimize both the background level and the thermal load by reflecting from UV to IR except over the $760-810 \mathrm{~nm}$ wavelength range

- NBF to reduce the bandwidth in the $777.4 \mathrm{I}-777.75 \mathrm{~nm}$ range of the lightning spectral pulse to improve the $\mathrm{S} / \mathrm{N}$ ratio

- For the SRW, a two-sided design approach was adopted: space side reflects the IR and Instrument side reflects the visible

- For the NBF, a multi-cavity Fabry Perot structure was used and a uniformity $< \pm 0.02 \%$ is typically achieved over a diameter of $120 \mathrm{~mm}$

- The NBF is annealed to fine-tune the CWL to within $10 \mathrm{pm}$ of the target

- Durability is verified through a comprehensive series of environmental tests, including radiation exposures of proton, Gamma, UV solar and electrons

- The SRW and NBF fully meets all the requirements of the Lightning Imager

www.facebook.com/IridianSpectralTechnologies/

in ca.linkedin.com/company/iridian-spectral-technologies

\section{$\underline{\text { twitter.com/IridianSpectral }}$}

www.iridian.ca/feed/ 